\title{
Arginine, glycine, aspartic acid peptide-modified paclitaxel and curcumin co-loaded liposome for the treatment of lung cancer: in vitro/vivo evaluation
}

This article was published in the following Dove Press journal: International Journal of Nanomedicine

\author{
Kanqiu Jiang* \\ Mingjing Shen* \\ Weihua Xu
}

Department of Cardiothoracic Surgery, The Second Affiliated Hospital of Soochow University, Suzhou Shi, Jiangsu Sheng, People's Republic of China

*These authors contributed equally to this work
Correspondence: Weihua Xu Province, 215004, People's Republic of China

Tel/fax +8651267784779

Email hwadoctor@126.com Sanxiang Road No 1055, Suzhou, Jiangsu

Purpose: In this study, a novel arginine, glycine, aspartic acid peptide (RGD)-modified paclitaxel and curcumin co-loaded liposomes were developed to evaluate their antitumor activity in vitro and in vivo.

Materials and methods: Co-loaded liposomes were prepared using the solvent evaporation method. The particles had spherical shapes under electron microscopy with sizes $<130 \mathrm{~nm}$.

Results: By comparison with the free drug, RGD-modified paclitaxel and curcumin co-loaded liposomes and paclitaxel and curcumin co-loaded liposomes have sustained-release properties in vitro. In vivo, there was no significant difference in pharmacokinetic parameters between the RGD-modified paclitaxel and curcumin co-loaded liposomes and paclitaxel and curcumin co-loaded liposomes. A strong green fluorescence was observed in the cytoplasmic region after incubation of RGD-modified paclitaxel and curcumin co-loaded liposomes for $2 \mathrm{~h}$. RGDmodified paclitaxel and curcumin co-loaded liposomes showed a superior antiproliferative effect on A549 cells with a possible mechanism that suppressed the multidrug resistance phenomenon and exhibited a clear synergistic effect.

Conclusion: The results indicate that RGD-modified paclitaxel and curcumin co-loaded liposomes had a better antitumor effect in vivo than the non-modified LPs. These results indicate that RGD-modified co-loaded liposomes are a promising candidate for antitumor drug delivery.

Keywords: arginine, glycine, aspartic acid peptide, paclitaxel, curcumin, liposome, cell uptake, cytotoxicity study, in vivo anti-tumor study

\section{Introduction}

Paclitaxel (Figure 1) (PTX), the prototype of taxanes agents, emerges from a natural source. ${ }^{1}$ Paclitaxel was effective in the treatment of a wide range of advanced human cancers, including non-small-cell lung cancer (NSCLC). ${ }^{2}$ The commercial PTX preparation $\left(\right.$ Taxol $^{\circledR}$ ) is formulated by Cremophor $\mathrm{EL}^{\circledR}$ (polyethoxylated castor oil used as a solubilizing agent) and anhydrous ethanol, which provides a homogeneous preparation. In clinical practice, PTX is usually administered as a $3-\mathrm{h}$ and $24-\mathrm{h}$ infusion representing a total dose of $135-175 \mathrm{mg} / \mathrm{m}^{2}$ of the body every 3 weeks. ${ }^{3}$ Although paclitaxel is the first choice for the treatment of lung cancer, its use is limited due to the limited uptake of the drug by p-gp. ${ }^{4}$

Curcumin (Figure 1B) (CUR) is a yellow polyphenol extracted from the rhizome of turmeric (Curcuma longa), a plant grown in tropical Southeast Asia. ${ }^{5}$ Enthusiasm for CUR as an anti-cancer agent was based on a larger number of epidemiological evidence, suggesting that dietary turmeric is associated with a lower incidence of gastrointestinal mucosal cancers. ${ }^{6,7}$ Numerous experimental data clearly indicate that free CUR 


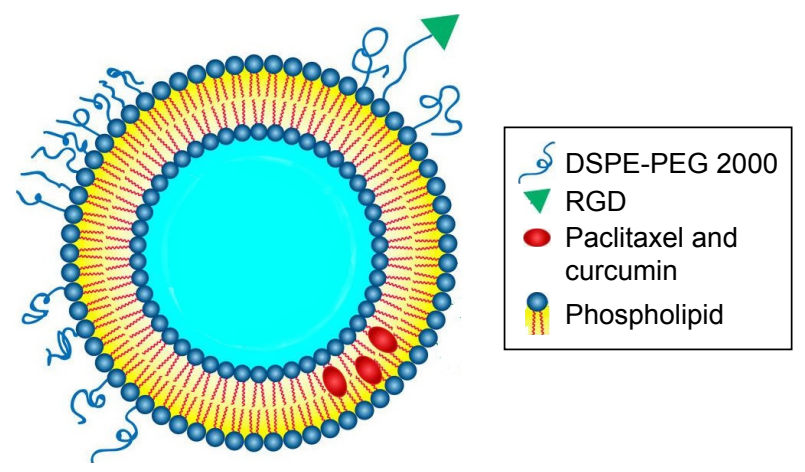

Figure I The microstructural schematic diagram of RGD-PTX-CUR LPs. Abbreviations: DSPE-PEG, distearoyl-L-a-phosphatidylethanolamine; PTX, paclitaxel; CUR, curcumin; LPs, liposomes; RGD, arginine, glycine, aspartic acid peptide.

induces cell cycle arrest and/or apoptosis in human cancer cell lines derived from a variety of solid tumors including colorectal, lung, breast, pancreatic and prostate carcinoma, and so on. ${ }^{8,9}$ In addition, some studies have shown that, after the reduction of PI3K/Akt and NF-kB pathways, CUR could independently display p-gp inhibitory activity. ${ }^{10,11}$ Therefore, CUR could be used as an effective p-gp inhibitor to maximize the cytotoxicity of anticancer drugs through combination with an anticancer drug on MDR-expressing cancer cells.

Despite PTX and CUR's multiple medicinal benefits, low solubility and bioavailability of both continues to be highlighted as a major challenge in developing formulations for clinical efficacy. Current improvements are mainly focused on the development of formulations that are devoid of toxic solvent, surveys of the potential for large-scale preparation, and requirements for long-term stability. Liposomes represent versatile and advanced nanoscale delivery systems for a wide range of bioactive compounds. ${ }^{12}$

These relatively non-toxic systems have a considerable potential to entrap both hydrophobic and hydrophilic drugs. Traditional common delivery systems have some disadvantages, such as inappropriate release profiles, and the use of conventional p-gp inhibitors to be toxic and non-targeted to normal cells. Therefore, the ideal composite delivery system should have a target feature and an optimal release profile. In other words, the exposure of anticancer agents and p-gp inhibitors to normal cells or tissues should be minimized and limited to specific target cells or tissues. ${ }^{13,14}$ The integrin receptors, especially $\alpha v \beta 3$, are often highly expressed in certain types of tumor cells and tumor vascular endothelial cells, but not in normal vessels. ${ }^{15}$ RGD is a short peptide containing arginine, glycine, and aspartic acid, which is widely found in vivo. RGD peptides, as the recognition site of integrin and its ligand, had adhesion between cells and extracellular matrix.
The exogenous RGD peptide has a competitive inhibitory ligand to integrins and inhibits angiogenesis and migration of tumor cells. At the same time, the tumor can be targeted, labeled, and anticancer drugs can be target delivered. ${ }^{16-18}$

In this experiment, we used liposomes (LPs) as a carrier, PTX and CUR as model drugs to prepare RGD-modified PTX-CUR LPs. LPs have good biodegradability and biocompatibility, and can achieve a sustained-release effect by gradually degrading in vivo. RGD peptide-modified LPs could improve the targeting ability of LPs. In addition, through enhanced permeability and retention (EPR) effects, it can increase the number of residual LPs in the tumor. ${ }^{19}$ In order to verify these properties of the LPs, various properties of RGD modified PTX-CUR LPs were studied in detail. Although there have been many reports on the delivery system of loading both PTX and CUR recently, ${ }^{20,21}$ the strategy of RGD modified was reported for the first time.

\section{Materials and methods Materials}

PTX and CUR was gifted from Yangzi River Pharmaceuticals Co Ltd (Jiangsu, People's Republic of China). Distearoyl-La-phosphatidylethanolamine (DSPE)-PEG2000 was provided by the FanShuo Biopharma Ltd (Shanghai, People's Republic of China). High purity cholesterol (CHOL) and hydrogenated soybean phosphatidyl choline (SPC) were purchased by Phospholipid Tech Ltd, Shanghai, People's Republic of China. RGD peptide was purchased from Enzo Life Sciences (Farmingdale, NY, USA). A549 lung cancer cell lines were purchased from the Institute of Biochemistry and Cell Biology (Shanghai, People's Republic of China). The chemical and solvents used were analytical or high performance liquid chromatography. Deionized water was prepared in the laboratory.

\section{Preparation of RGD-modified PTX-CUR LPS}

RGD-modified PTX and CUR co-loaded LPs were prepared using the solvent evaporation method. ${ }^{22}$ Briefly, PTX $(5 \mathrm{mg})$, CUR (3 mg), CHOL (15 mg), DSPE-PEG 2000 (12.5 mg) and SPC (120 mg) were dissolved in $10 \mathrm{~mL}$ of chloroform to produce an oil phase. This was attached to a rotary evaporator and the organic phase was removed by evaporating at $45^{\circ} \mathrm{C} \pm 2^{\circ} \mathrm{C}$, which led to the formation of the film on the wall of the flask. The flask remained overnight to remove any trace in the solvent. The lipid membranes were hydrated with $5 \mathrm{~mL}$ of phosphate-buffered saline (PBS, $\mathrm{pH} 7.4$, containing $50 \mathrm{mg}$ RGD peptide) at $37^{\circ} \mathrm{C}$ for $30 \mathrm{~min}$. The suspension was then subjected to ultrasonic treatment for $2 \mathrm{~min}$ at $500 \mathrm{~W}$ using a 
high-intensity probe ultrasonicator (JY92-2D; Xinzhi Equipment Instruction, Zhejiang, People's Republic of China). The same method was used to prepare PTX and CUR co-loaded LPs without RGD peptide as the control samples.

\section{Characterization}

Morphological examination of LPs was performed using Philips CM120 TEM (FEI, Eindhoven, the Netherlands). In practice, the LPs solution containing $0.1 \%(\mathrm{w} / \mathrm{v})$ phosphotungstic acid was placed on the carbon film, and was observed by electron microscope at $80 \mathrm{kV}$.

A NICOMP 380 Submicron Particle Sizer with dynamic light scattering (DLS, PSS company, Santa Barbara, USA) was used to determine the particle size distribution and mean diameter of the prepared RGD-modified PTX-CUR LPs. Sample solutions were transferred into the light scattering cells. The intensity autocorrelation was measured at a scattering angle of $90^{\circ}$ at room temperature. Data was analyzed in terms of intensity-weighted NICOMP distributions. The experimental results of each report are obtained with an average autocorrelation function of at least three $\mathrm{DH}$ values accumulated at least $20 \mathrm{~min}$ from the analysis. Zeta potential was measured on the same samples prepared for size analysis.

\section{Characterization of LPs}

Drug-loading coefficient (DL\%) and encapsulation ratio (ER\%) were calculated as described previously. ${ }^{23,24}$ Firstly, PTX and CUR were extracted from the LPs with $1 \mathrm{~mL}$ $2 \%$ acetic acid: acetonitrile $(1: 1, \mathrm{v} / \mathrm{v})$, and then the extract solution was properly diluted prior to HPLC analysis. The content of PTX and CUR in the LPs was determined by the HPLC method described below. Then, DL\% and ER\% were calculated according to $\mathrm{Eq}(1)$ and $\mathrm{Eq}(2)$ :

$$
\begin{gathered}
\mathrm{DL} \%=\frac{\mathrm{W}_{\mathrm{M}}}{\left(\mathrm{W}_{\mathrm{P}}+\mathrm{W}_{\mathrm{M}}\right)} \times 100 \\
\mathrm{ER} \%=\frac{\mathrm{W}_{\mathrm{M}}}{\mathrm{W}_{\mathrm{F}}} \times 100
\end{gathered}
$$

where $\mathrm{W}_{\mathrm{P}}$ is the weight of the initial feeding polymer, $\mathrm{W}_{\mathrm{M}}$ is the weight of the drug incorporated in LPs, and $W_{F}$ is the weight of the initial feeding drug.

\section{In vitro drug release}

In vitro release of CUR from the LPs was carried out by the dialysis membrane method. RGD-modified PTX and CUR co-loaded LPs ( $\approx 5 \mathrm{mg}$ PTX and $3 \mathrm{mg}$ CUR) were re-dispersed in $1 \mathrm{~mL}$ of deionized water in dialysis bags (sigma) with a molecular cut off of $10 \mathrm{kDa}$. The bags were suspended in the release of $18 \mathrm{~mL}$ medium (ethanol 50\%, v/v) while shaking in a $37^{\circ} \mathrm{C}$ water bath at $75 \mathrm{rpm}$. The $0.1 \mathrm{~mL}$ sample was extracted at the predetermined time interval and replaced with fresh medium. Finally, the samples were analyzed by HPLC. In the release medium, ethanol was used to provide the sink condition because PTX and CUR were insoluble in water.

The control PTX and CUR co-loaded LPs without RGD peptide were prepared using the same method.

\section{Stability study}

Accelerated stability studies were conducted under the International Conference on Harmonisation (ICH) guidelines (1993). Sealed bottles of freshly prepared freeze-dried LPs were placed in a stability chamber maintained at $25^{\circ} \mathrm{C}$, $60 \%$ relative humidity $(\mathrm{RH})$. The physical appearance, size, physical and chemical properties of the samples were analyzed over a 3-month period and with a frequency of 1-month sampling.

\section{Pharmacokinetics}

All the in vivo experimental protocols were approved by the animal care committee of Soochow University and all experiments were conducted in strict accordance with the laboratory animal care and use guidelines adopted by the National Institutes of Health (Shanghai, People's Republic of China). A total of 30 Sprague Daley rats (half male) weighing from 180 to $220 \mathrm{~g}$ were used for the pharmacokinetics study. Before the experiment commenced, the rats were kept in a state of fasting for $6 \mathrm{~h}$. All animals were randomly divided into three groups with 10 in each group via iv injection of PTX and CUR suspension, conventional PTX/CUR LPs, and RGD-PTX/CUR LPs. The doses of PTX and CUR in the three groups were 10 and $6 \mathrm{mg} / \mathrm{kg}$, respectively. Blood samples $(2 \mathrm{~mL})$ were collected from the tail vein into heparinized $5 \mathrm{~mL}$ polythene tubes just before administration and $0.25,0.5,1,2,4,6,8,10,12$ and $24 \mathrm{~h}$ after dosing. The plasma obtained was stored at $-20^{\circ} \mathrm{C}$ until analysis. The sample handling and determination process was referenced to previous literature by the LC-MS/MS method. ${ }^{25-28}$

\section{Cell uptake}

The cellular internalization of free PTX/CUR, PTX/CUR LPs and RGD-PTX/CUR LPs was observed with confocal microscopy by using coumarin- 6 as a fluorescent probe. A549 cells were grown in RPMI 1640 medium with 10\% (v/v) 
FBS and $5 \%$ antibiotics (100 IU/mL of penicillin G sodium and $100 \mu \mathrm{g} / \mathrm{mL}$ of streptomycin sulfate). A549 cells were inoculated at the initial density of $4 \times 10^{5}$ cells per dish. Cells were then incubated with coumarin-6-adsorbed free PTX/ CUR, PTX/CUR LPs and RGD-PTX/CUR LPs (equivalent to $0.1 \mu \mathrm{g} / \mathrm{mL}$ of coumarin-6) for $2 \mathrm{~h}$ at $37^{\circ} \mathrm{C} \pm 0.5^{\circ} \mathrm{C}$.

Subsequently, the cells were washed with PBS and fixed with 4\% paraformaldehyde for 10 min under confocal microscopy. For PTX/CUR uptake quantitative estimates, the density of cells inoculated on 24-well plates was $3 \times 10^{4}$ cells. When reaching a confluence of $70 \%-80 \%$, the cells were incubated with coumarin-6 adsorbed free PTX/ CUR, PTX/CUR LPs and RGD-PTX/CUR LPs (equivalent to $0.1 \mu \mathrm{g} / \mathrm{mL}$ of coumarin-6). After $2 \mathrm{~h}$ of incubation, the cells were washed several times with cold PBS and then dissolved with Triton X-100 (0.1\%). Multimode microplate reader was used to measure the fluorescence intensity and the excitation/emission wavelength was 440 and $520 \mathrm{~nm}$, respectively.

\section{Cytotoxicity study}

The cytotoxicity of blank LPs, free PTX/CUR, PTX/CUR LPs and RGD-PTX/CUR LPs was evaluated in A549 cells by MTT assay. Briefly, A549 cells were seeded in a 96-well plate at a density of 3 to $4 \times 10^{3}$ cells per well. After $12 \mathrm{~h}$, different PTX/CUR formulations (PTX/CUR concentrations ranging from 0.0025 to $25 \mu \mathrm{g} / \mathrm{mL}$ were added, and plates were incubated for 24 and 48 h, PTX:CUR=5:3). PTX/CUR standard solution was prepared with PTX/CUR dissolved in ethanol ranging from 0.25 to $2.5 \mathrm{mg} / \mathrm{mL}$ and then diluted 100 times with distilled water. Measurements were taken using a microplate reader.

\section{In vivo anti-tumor study}

The antitumor effect of RGD-PTX/CUR LPs was evaluated in vivo using BALB/c nude mice (aged 5-6 weeks, weight 20-22 g) that had been inoculated subcutaneously with $2 \times 10^{9}$ human lung adenocarcinoma (A549) cells. The treatments were started on the day when the tumor volume reached $100-150 \mathrm{~mm}^{3}$, which was designated as day 0 . On the 8 th day, the mice were randomly assigned to four groups (6 animals per group): group 1 was given a 5\% glucose injection, group 2 was given free PTX and CUR, group 3 was given PTX/CUR LPs and group 4 was given RGD-PTX/ CUR LPs. All the formulations were given via the tail vein on days $8,10,12$, and 14 , at a dose of $10 \mathrm{mg} / \mathrm{kg}$ (PTX) and $6 \mathrm{mg} / \mathrm{kg}$ (CUR). A digital caliper was used to measure the tumor diameters and tumor volumes $\left(\mathrm{mm}^{3}\right)$ were calculated by the following formula: tumor volume $=$ length $\times$ width ${ }^{2} \times 0.5$. Throughout the study, mice were weighed regularly to monitor the potential toxicities.

\section{Statistical analysis}

All experiments were expressed as mean \pm SD and performed in triplicate. Statistical analysis was carried out by ANOVA analysis using GraphPad Prism 6.0. Statistically significant change is considered at $p<0.05$.

\section{Results and discussion Preparation and characterization}

A solvent evaporation method was employed to prepare RGD-modified PTX and CUR co-loaded LPs and the method was simple and easy to scale up. In the process, lipid solution was first prepared by dissolving solid lipids with chloroform. The resulting lipid film was hydrated phosphate-buffered saline which was then filtered and dried. As shown in Figure 2, the surface morphology of PTX and CUR LPs was observed by TEM. The LPs were spherical in shape with a smooth surface and the size was uniform and appropriate for administration via intravenous injection. The mean diameter of RGD-PTX/CUR LPs was $120.6 \pm 10.8 \mathrm{~nm}$ and the poly disperse index (PDI) was $0.139 \pm 0.026$. The other parameters
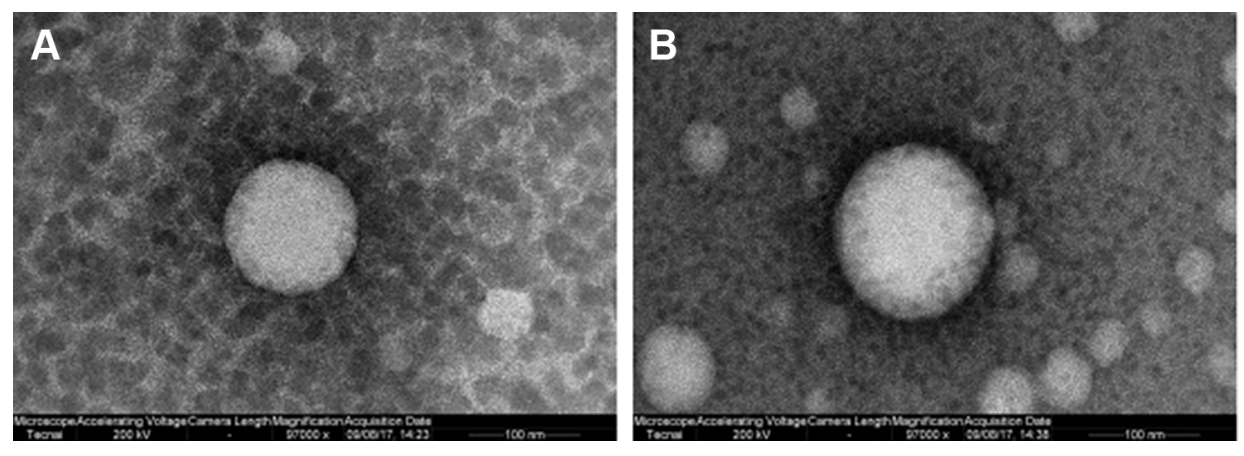

Figure 2 Transmission electron microscope photograph of PTX and CUR co-loaded LPs.

Note: (A) Not RGD modified, (B) RGD modified (magnification $\times 97,000$ ).

Abbreviations: PTX, paclitaxel; CUR, curcumin; LPs, liposomes; RGD, arginine, glycine, aspartic acid peptide. 
Table I Characterization of the prepared LPs

\begin{tabular}{|c|c|c|c|c|c|c|c|}
\hline \multirow[t]{2}{*}{ Formulation } & \multirow{2}{*}{$\begin{array}{l}\text { Mean diameter } \\
(\mathrm{nm})\end{array}$} & \multirow[t]{2}{*}{ PDI } & \multirow{2}{*}{$\begin{array}{l}\text { Zeta potential } \\
(\mathrm{mV})\end{array}$} & \multicolumn{2}{|l|}{ PTX } & \multicolumn{2}{|l|}{ CUR } \\
\hline & & & & DL\% & ER\% & DL\% & ER\% \\
\hline RGD-PTX/CUR LPs & $120.6 \pm 10.83$ & $0.139 \pm 0.026$ & $-5.62 \pm 1.13$ & $7.6 \pm 2.13$ & $85.3 \pm 6.72$ & $8.6 \pm 1.72$ & $82.3 \pm 7.31$ \\
\hline PTX/CUR LPs & $117.2 \pm 8.43$ & $0.142 \pm 0.035$ & $-6.25 \pm 1.69$ & $7.2 \pm 3.21$ & $76.5 \pm 6.24$ & $8.7 \pm 1.65$ & $83.9 \pm 6.82$ \\
\hline
\end{tabular}

Abbreviations: PDI, poly disperse index; PTX, paclitaxel; CUR, curcumin; DL\%, drug-loading coefficient; ER\%, encapsulation ratio; RGD, arginine, glycine, aspartic acid peptide; LPs, liposomes.

are shown in Table 1. Obviously, there were no significant differences in the parameters between the modified liposomes and the unmodified liposomes. In addition, the zeta potential of liposomes indicates that there was a number of negative charges on the surface, which makes the whole system have good physical stability. There were no significant changes in the indexes after adding RGD into liposomes.

\section{In vitro drug release}

The in vitro cumulative drug-release profile is shown in Figure 3. Over time, both PTX and CUR in LPs was released much more slowly than the free drug. At $2 \mathrm{~h}$, the cumulative release of the free drug (PTX) was about $90 \%$ but about $40 \%$ in LPs (CUR 86\%, 30\%). A biphasic release was observed in this case with a rapid release of about $40 \%$ in $2 \mathrm{~h}$ followed by sustained drug release of about $75 \%$ over $12 \mathrm{~h}$ (CUR 30\%, 60\%). This suggests that in comparison with the free drug, RGD-PTX-CUR LPs and PTX-CUR LPs have sustained-release properties. However, there was no significant difference between the two LPs in the release curve. Phospholipid, which is the main ingredient used in this experiment, is a corrosive substance. Because of the
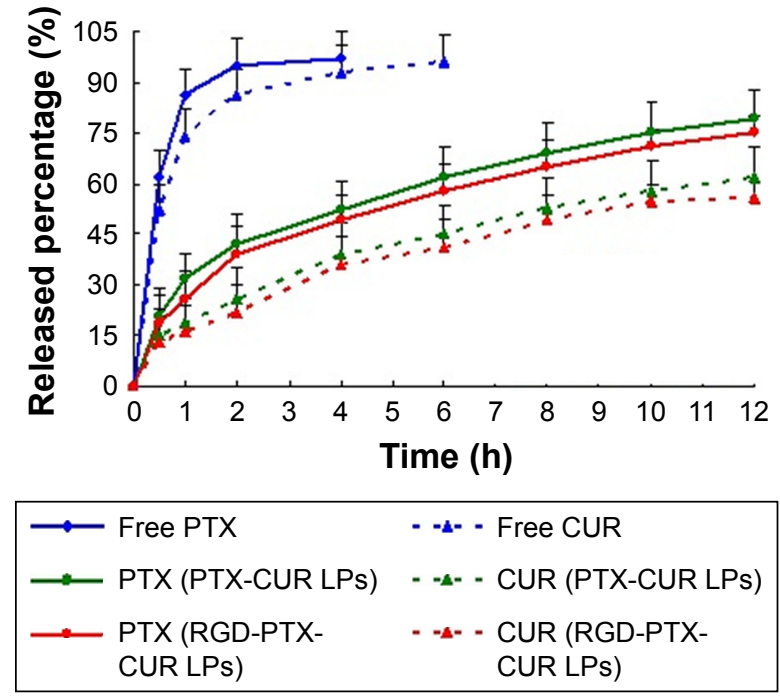

Figure 3 The release profile of free PTX, free CUR, PTX-CUR LPs and RGD-PTXCUR LPs from release medium $\left(50 \%, v / v\right.$, of ethanol) at $37^{\circ} \mathrm{C}$ in a shaking water bath at $75 \mathrm{rpm}(\mathrm{n}=6)$.

Abbreviations: PTX, paclitaxel; CUR, curcumin; LPs, liposomes; RGD, arginine, glycine, aspartic acid peptide. poor water solubility of this drug, it is difficult for it to penetrate into the matrix of the LPs. The sustained release of the drug might mainly contribute to the corrosion rates of the LPs prepared with the phospholipid. After calculation, the in vitro drug-release kinetic model of PTX and CUR (RGD-PTX-CUR LPs) in release medium fit well with the Higuchi equation: $\mathrm{Q}=6.595 \mathrm{t} 1 / 2+0.652$ (PTX, $\mathrm{r}=0.996$ ) and $\mathrm{Q}=3.597 \mathrm{t} 1 / 2-0.659$ (CUR, $\mathrm{r}=0.995$ ). Thus, it was speculated that the sustained-release property of LPs could enhance absorption of PTX and CUR.

\section{Stability study}

RGD-modified PTX and CUR co-loaded LPs exhibited good stability over the period of 3 months. No significant change in physical appearance and particle aggregation was observed. Meanwhile LPs had a good physical stability. None of the indexes changed markedly during the observation period. Both PTX and CUR in the formulations were observed with no degradation (Table 2).

\section{Pharmacokinetics}

The profiles of PTX and CUR concentration in plasma vs time are shown in Figure 4. As is already known, the liposomal PTX or CUR formulation had a much longer systemic circulation time when compared with the suspension. Both PTX and CUR from suspensions were eliminated in vivo in a short time, and the concentration in plasma was lower than $20 \mathrm{ng} / \mathrm{mL}$ at $6 \mathrm{~h}$ after administration. Based on the analysis of the models and parameters with DAS2.0, it was concluded

Table 2 Physical stability of RGD-modified PTX and CUR co-loaded LPs at $25^{\circ} \mathrm{C}, 60 \% \mathrm{RH}(\mathrm{n}=3)$

\begin{tabular}{llll}
\hline Parameters & 0 day & 2 months & 3 months \\
\hline Physical appearance & Milk white & Milk white & Milk white \\
Particle size $(\mathrm{nm})$ & $120.6 \pm 10.83$ & $121.6 \pm 9.15$ & $123.5 \pm 7.33$ \\
Zeta potential $(\mathrm{mV})$ & $-5.62 \pm I .13$ & $-7.2 \pm 1.42$ & $-7.6 \pm 1.73$ \\
DL\% (PTX) & $7.6 \pm 2.13$ & $7.3 \pm 3.42$ & $7.2 \pm 4.23$ \\
ER\% (PTX) & $85.3 \pm 6.72$ & $83.5 \pm 8.34$ & $81.7 \pm 8.92$ \\
DL\% (CUR) & $8.6 \pm 1.72$ & $8.4 \pm I .56$ & $8.1 \pm 1.65$ \\
ER\% (CUR) & $82.3 \pm 7.31$ & $81.1 \pm 6.52$ & $80.9 \pm 5.32$ \\
\hline
\end{tabular}

Abbreviations: RGD, arginine, glycine, aspartic acid peptide; PTX, paclitaxel; CUR, curcumin; LPs, liposomes; RH, relative humidity; DL\%, drug-loading coefficient; ER\%, encapsulation ratio. 

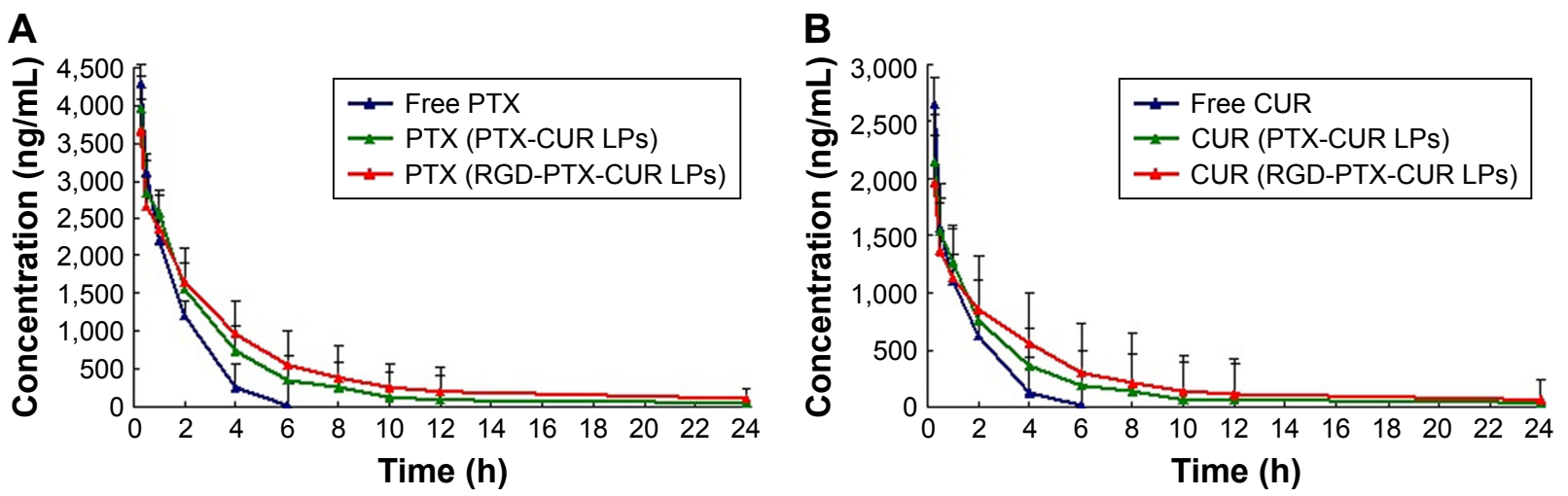

Figure 4 Plasma concentration-time profiles.

Note: (A) PTX and (B) CUR after iv administration of different formulations to rats $(n=10)$.

Abbreviations: PTX, paclitaxel; CUR, curcumin; LPs, liposomes; RGD, arginine, glycine, aspartic acid peptide.

that the in vivo pharmacokinetics of RGD-PTX/CUR LPs in blood could be described by a two-compartment model after iv administration. In this study, rats treated with RGD-PTX/ CUR LPs had a slightly higher plasma concentration than those treated with PTX/CUR LPs. The pharmacokinetic parameters are reported in Table 3. As shown in Table 3, the pharmacokinetic parameters of RGD-PTX/CUR LPs in rats showed changes in comparison to the other two groups. The mean residence time was significantly higher than that of the suspension. The RGD-PTX/CUR LPs provided a higher $\mathrm{AUC}_{0-\infty}$ (PTX:1.16-fold; CUR:1.23-fold), mean residence time (PTX:1.13-fold; CUR:1.39-fold), and biological halflife (PTX:1.17-fold; CUR:1.34-fold) when compared with PTX/CUR LPs. However, the RGD-PTX/CUR LPs showed decreased clearance when compared with the PTX/CUR LPs. There was no significant difference in pharmacokinetic parameters between the RGD-PTX/CUR LPs and PTX/CUR LPs. Usually, a hydrophilic surface-modified nano-carrier can be prevented by the reticuloendothelial system, have long circulating action, and change the pharmacokinetics. In the present study, hydrophobic RGD was coupled to PTX/CUR
LPs and extended the circulation of PTX and CUR. The variation of AUC indicated that the RGD-PTX/CUR LPs provided higher bioavailability than the PTX/CUR suspension.

\section{Cell uptake}

Confocal microscopy was employed to characterize the cellular internalization of free PTX/CUR, PTX/CUR LPs and RGD-PTX/CUR LPs in A549 cells. As shown in Figure 5, a strong green fluorescence was observed in the cytoplasmic region of RGD-PTX/CUR LPs after incubation for $2 \mathrm{~h}$. In A549 cells, the cell internalization of RGD-PTX/CUR LPs was higher than others. The results showed that surface modification could indicate when cell internalization was changed and more drugs entered the cells successfully. In quantitative cell uptake studies, coumarin-6 in the three formulations was quantified by recovering the drug LPs from cells and measuring their fluorescence (normalized to per mg of the total cellular protein contents). Quantitative results were consistent with the confocal images (data not shown).

Table 3 Pharmacokinetic parameters of drug after iv administration of LPs and suspensions to rats $(n=10)$

\begin{tabular}{|c|c|c|c|c|c|c|}
\hline \multirow[t]{2}{*}{ Parameters } & \multicolumn{3}{|l|}{ PTX } & \multicolumn{3}{|l|}{ CUR } \\
\hline & Free drug & $\begin{array}{l}\text { PTX/CUR } \\
\text { LPs }\end{array}$ & $\begin{array}{l}\text { RGD-PTX/CUR } \\
\text { LPs }\end{array}$ & Free drug & $\begin{array}{l}\text { PTX/CUR } \\
\text { LPs }\end{array}$ & $\begin{array}{l}\text { RGD-PTX/ } \\
\text { CUR LPs }\end{array}$ \\
\hline$t_{1 / 2}(h)$ & $5.4 \pm 1.4$ & $11.5 \pm 2.2$ & $13.5 \pm 2.6^{*}$ & $3.6 \pm I .4$ & $6.1 \pm 1.1$ & $8.2 \pm 2.1^{*}$ \\
\hline$A \cup C_{0-t}(n g \cdot h / m L)$ & $6,256.3 \pm 6 \mid 2.7$ & $10,335.3 \pm 983.2$ & $12,760.4 \pm 1,102 . I^{*}$ & $3,286.5 \pm 356.2$ & $5,321.9 \pm 526.3$ & $6,654.9 \pm 625.4^{*}$ \\
\hline$A \cup C_{0-\infty}(\mathrm{ng} \cdot \mathrm{h} / \mathrm{mL})$ & $7,105.4 \pm 659.3$ & $|2,3| 5.2 \pm 9 \mid 6.3$ & $|4,25| .3 \pm 1,0 \mid 4.5^{*}$ & $3,956.2 \pm 4 \mid 2.2$ & $6,123.4 \pm 598.4$ & $7,536.7 \pm 7 \mid 4.3 *$ \\
\hline MRT (h) & $6.1 \pm 1.7$ & $15.3 \pm 2.6$ & $17.3 \pm 3.1^{*}$ & $4.8 \pm 1.3$ & $8.2 \pm 1.8$ & $\mid I .4 \pm 2.1 *$ \\
\hline$C L(L / h)$ & $13.8 \pm 2.9$ & $6.9 \pm 1.8$ & $4.9 \pm 1.3^{*}$ & $11.2 \pm 1.2$ & $6.2 \pm 1.1$ & $4.1 \pm 1.2 *$ \\
\hline
\end{tabular}

Note: $*_{p}<0.05:$ RGD-PTX/CUR LPs and Free drug (PTX or CUR).

Abbreviations: PTX, paclitaxel; CUR, curcumin; RGD, arginine, glycine, aspartic acid peptide; LPs, liposomes; $t_{1 / 2}$, half-life time; MRT, mean residence time; CL, clearance. 

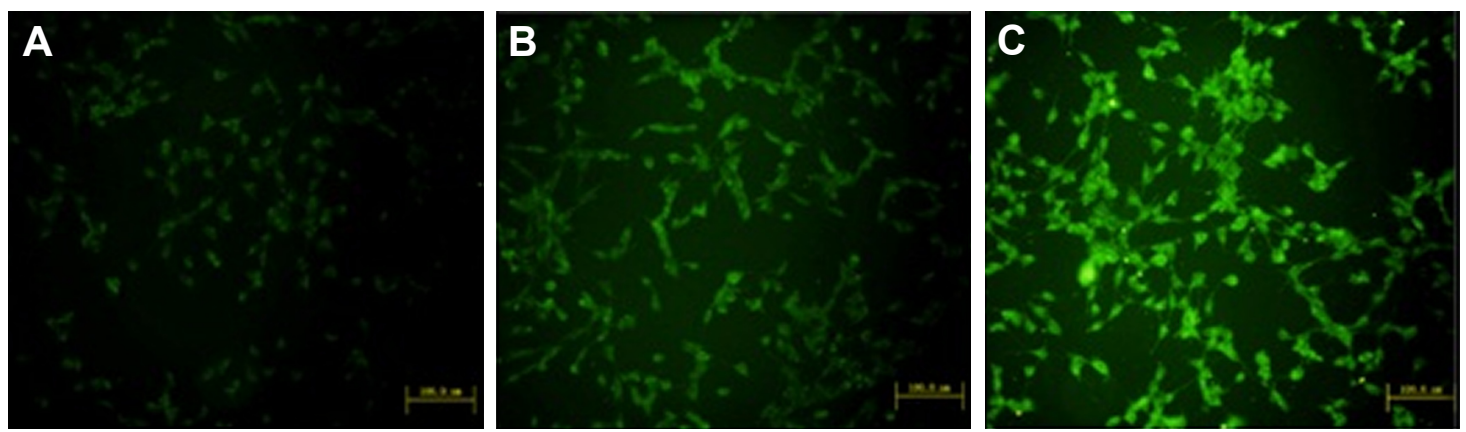

Figure 5 Confocal images of cellular uptake.

Notes: (A) Free PTX/CUR, (B) PTX/CUR LPs, and (C) RGD-PTX/CUR LPs by 549 cells. Incubation time was $2 \mathrm{~h}$.

Abbreviations: PTX, paclitaxel; CUR, curcumin; LPs, liposomes; RGD, arginine, glycine, aspartic acid peptide.

\section{Cytotoxicity study}

The effects of different PTX preparations on A549 cells were detected by MTT assay (Figure 6). It can be seen that free PTX/CUR, PTX/CUR LPs and RGD-PTX/CUR LPs exhibited a time-dependent and dose-dependent cytotoxicity in the cell lines tested. Although PTX and CUR are powerful antitumor drugs, they do not completely inhibit cell proliferation. Similarly, the combination of LPs and PTX/CUR has no superior effect. On the other hand, RGD coupled with PTX/CUR significantly reduces cell proliferation and improved therapeutic efficiency. Surprisingly, RGD-PTX/ CUR LPs showed a higher antiproliferative effect on A549 cells, which may have a possible mechanism that inhibited multidrug resistance phenomenon and showed an obvious synergistic effect. All formulations values of half maximal inhibitory concentration (IC50) are listed in Table 4. The results showed that IC50 value decreased to different extents depending on incubation time, and RGD-PTX/CUR LPs have strong targeting ability, which can improve the cellular uptake and cytotoxic of PTX/CUR.

\section{In vivo tumor growth inhibition}

To test the antitumor activity of RGD-PTX/CUR LPs, mice bearing human lung adenocarcinoma (A549) tumors were treated with conventional LPs or RGD-modified LPs four times over 14 days. As shown in Figure 7, the two types of LPs inhibited growth of the A549 cells and reduced the size of the tumors compared with the control group. However, there was a significant decrease in tumor volume in the treatment group with RGD-PTX/CUR LPs and PTX/CUR LPs group with only a small decrease in tumor volume, when compared with the control group. The average weight and volume of the tumors are shown in Table 4. The appearance of tumors was consistent with the statistical analysis of tumor volume data. Compared with the control group, all the treatments were significant $(p<0.05)$, and the tumor volume was inhibited,
A

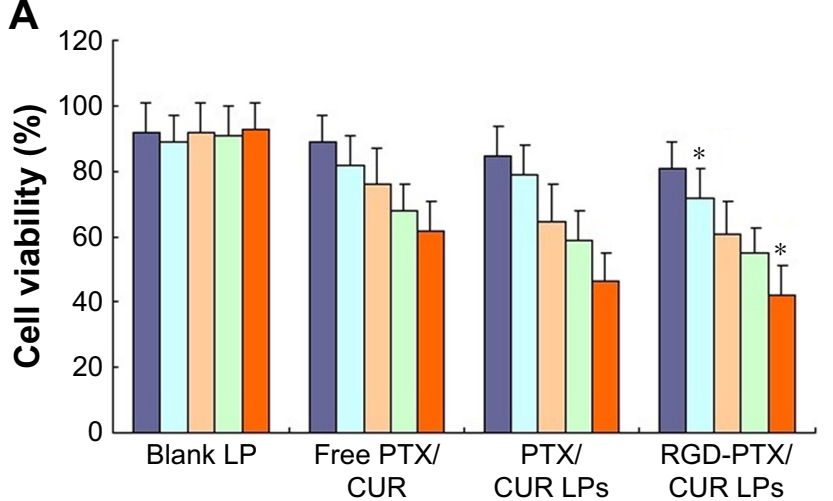

B

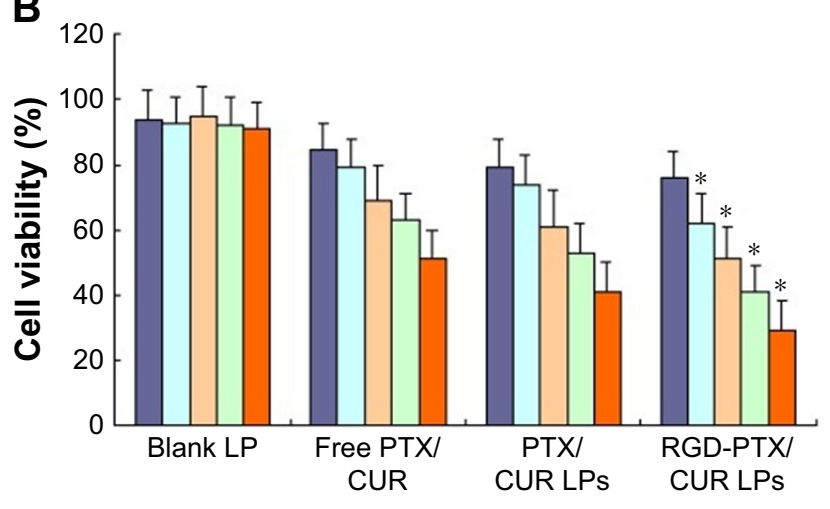

$0.0025 \mu \mathrm{g} / \mathrm{mL} \quad \square 0.025 \mu \mathrm{g} / \mathrm{mL} \quad \square 0.25 \mu \mathrm{g} / \mathrm{mL} \quad \square 2.5 \mu \mathrm{g} / \mathrm{mL} \quad \square 25 \mu \mathrm{g} / \mathrm{mL}$

Figure 6 In vitro cytotoxicity analysis of free PTX/CUR, PTX/CUR LPs and RGD-PTX/CUR LPs on A549 cell lines.

Notes: (A) 24 hours, or (B) 48 hours. Cell viability assay was performed by MTT assay. ${ }^{*}<<0.05$ RGD-PTX/CUR LPs vs PTX/CUR LPs.

Abbreviations: PTX, paclitaxel; CUR, curcumin; LPs, liposomes; RGD, arginine, glycine, aspartic acid peptide. 
Table 4 Effect of free PTX/CUR, PTX/CUR LPs and RGD-PTX/CUR LPs on A549 cells in nude mice $(n=12)$

\begin{tabular}{|c|c|c|c|c|c|}
\hline \multirow[t]{2}{*}{ Formulation } & \multicolumn{2}{|c|}{ TV $\left(\mathrm{mm}^{3}\right)$} & \multirow{2}{*}{$\begin{array}{l}\text { Tumor } \\
\text { weight (mg) }\end{array}$} & \multirow[t]{2}{*}{$\mathbf{R}$} & \multirow{2}{*}{$\begin{array}{l}\text { IR } \\
\text { (\%) }\end{array}$} \\
\hline & $d_{1}$ & $d_{16}$ & & & \\
\hline Control group & $132 \pm 26$ & $725 \pm 215$ & $748 \pm 215$ & $5.49 \pm 1.36$ & \\
\hline Free PTX/CUR & $134 \pm 28$ & $269 \pm 124$ & $297 \pm 165$ & $2.01 \pm 1.12$ & 63.4 \\
\hline PTX/CUR LPs & $135 \pm 24$ & $232 \pm 119$ & $229 \pm 124$ & $1.72 \pm 0.8 \mid$ & 68.7 \\
\hline RGD-PTX/CUR LPs & $|37 \pm 3|$ & $89 \pm 26^{a, b, c}$ & $95 \pm 32^{a, b, c}$ & $0.65 \pm 0.29 \mathrm{a}, \mathrm{b}, \mathrm{c}$ & 88.2 \\
\hline
\end{tabular}

Notes: ${ }^{a} p<0.01$ vs control group; ${ }^{b} p<0.05$ vs free PTX/CUR group; ${ }^{c} p<0.05$ vs PTX/CUR LPs.

Abbreviations: PTX, paclitaxel; CUR, curcumin; RGD, arginine, glycine, aspartic acid peptide; LPs, liposomes; TV, tumor volume; R, relative tumor volume; IR, inhibited rate.

and the results were the most effective in the RGD modification group. Similarly, the weight of the tumor was significant $(p<0.05)$, and was inhibited by both PTX/CUR treatments. Once again, the effect was most obvious in the RGD-PTX/ CUR LPs group. Interestingly, the data show that the tumor weight was more sensitive to treatment than the tumor volume. In general, our results show that the antitumor effect of RGD-PTX/CUR LPs was better than that of unmodified LPs in vivo. No significant pathological changes were found in any of the treatment groups (data not shown).

\section{Conclusion}

A novel RGD-modified paclitaxel and curcumin co-loaded liposomes were developed by using the solvent evaporation
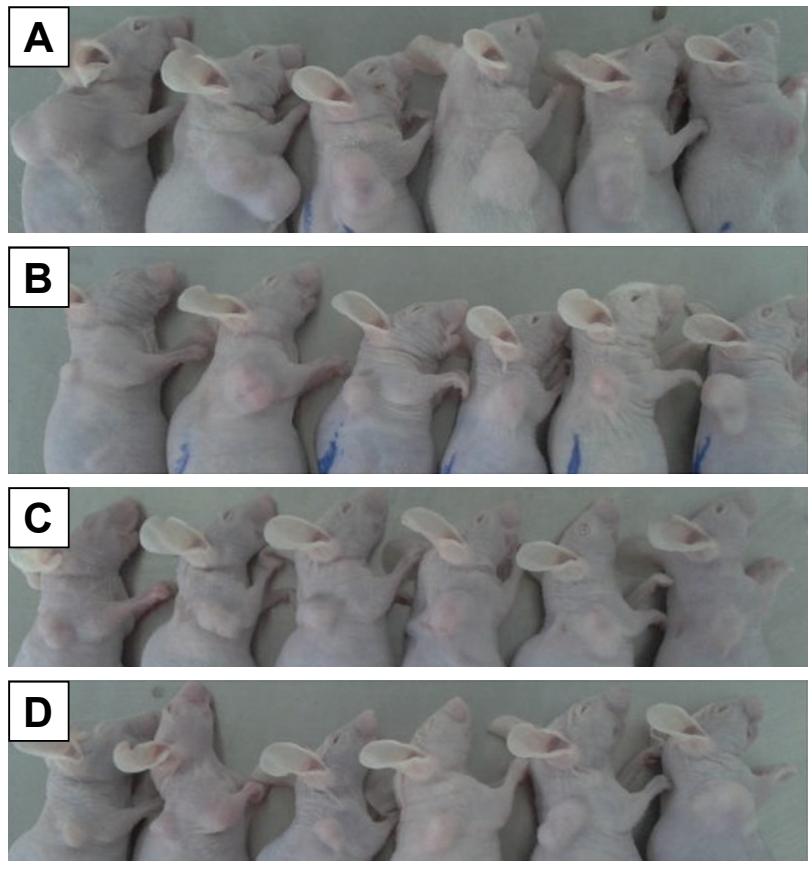

Figure 7 The tumor volume in nude mice transplanted with a human adenocarcinoma (A549) cell line on day 16.

Note: (A) Control group, (B) free PTX/CUR, (C) PTX/CUR LPs and (D) RGDPTX/CUR LPs.

Abbreviations: PTX, paclitaxel; CUR, curcumin; LPs, liposomes; RGD, arginine, glycine, aspartic acid peptide. method. In comparison with the free drug, RGD-PTX-CUR LPs and PTX-CUR LPs have sustained-release properties in vitro release. In vivo, there was no significant difference in pharmacokinetic parameters between the RGD-PTX/CUR LPs and PTX/CUR LPs. A strong green fluorescence was observed in the cytoplasmic region after incubation of RGDPTX/CUR LPs for $2 \mathrm{~h}$. RGD-PTX/CUR LPs showed a higher antiproliferative effect on A549 cells, which may be a possible mechanism that inhibited multidrug resistance phenomenon and showed an obvious synergistic effect. Finally, the results indicate that RGD-PTX/CUR LPs had a better antitumor effect in vivo than the non-modified LPs. These results indicate that RGD-modified co-loaded liposomes were a promising candidate for antitumor drug delivery.

\section{Acknowledgments}

This study was funded by Suzhou science and technology item (SYS201719) and (SYSD2016090).

\section{Disclosure}

The authors report no conflicts of interest in this work.

\section{References}

1. Stage TB, Bergmann TK, Kroetz DL. Clinical pharmacokinetics of paclitaxel monotherapy: an updated literature review. Clin Pharmacokinet. 2018;57(1):7-19.

2. Lu H, Jiang Z. Advances in antiangiogenic treatment of small-cell lung cancer. Onco Targets Ther. 2017;10:353-359.

3. Kramer I, Heuser A. Paclitaxel pharmaceutical and pharmacological issues. Eur Hosp Pharm. 1995;1:37-41.

4. Zhao M, Lei C, Yang Y, et al. Abraxane, the nanoparticle formulation of paclitaxel can induce drug resistance by up-regulation of p-gp. PLoS One. 2015;10(7):e0131429.

5. Shishodia S, Sethi G, Aggarwal BB. Curcumin: getting back to the roots. Ann N Y Acad Sci. 2005;1056:206-217.

6. Mohandas KM, Desai DC. Epidemiology of digestive tract cancers in India. V. Large and small bowel. Indian J Gastroenterol. 1999;18(3): $118-121$.

7. Sinha R, Anderson DE, McDonald SS, Greenwald P. Cancer risk and diet in India. $J$ Postgrad Med. 2003;49(3):222-228.

8. Khor TO, Keum YS, Lin W, et al. Combined inhibitory effects of curcumin and phenethyl isothiocyanate on the growth of human PC-3 prostate xenografts in immunodeficient mice. Cancer Res. 2006;66(2): 613-621. 
9. Maheshwari RK, Singh AK, Gaddipati J, Srimal RC. Multiple biological activities of curcumin: a short review. Life Sci. 2006;78(18): 2081-2087.

10. Choi BH, Kim CG, Lim Y, Shin SY, Lee YH. Curcumin down-regulates the multidrug-resistance mdr1b gene by inhibiting the PI3K/Akt/NF kappa B pathway. Cancer Lett. 2008;259(1):111-118.

11. Neerati P, Sudhakar YA, Kanwar JR. Curcumin regulates colon cancer by inhibiting p-glycoprotein in in-situ cancerous colon perfusion rat model. J Cancer Sci Ther. 2013;5:313-319.

12. Hofheinz RD, Gnad-Vogt SU, Beyer U, Hochhaus A. Liposomal encapsulated anticancer drugs. Anticancer Drugs. 2005;16(7):691-707.

13. Wessler JD, Grip LT, Mendell J, Giugliano RP. The P-glycoprotein transport system and cardiovascular drugs. J Am Coll Cardiol. 2013; 61(25):2495-2502.

14. Thadakapally R, Aafreen A, Aukunuru J, Habibuddin M, Joqala S. Preparation and characterization of PEG albumin-curcumin nanoparticles intended to treat breast cancer. Indian J Pharm Sci. 2016;78(1): 65-72.

15. Zetter BR. On target with tumor blood vessel markers. Nat Biotechnol. 1997;15(12):1243-1244.

16. Danhier F, Le Breton A, Préat V. RGD-based strategies to target alpha(v) beta(3) integrin in cancer therapy and diagnosis. Mol Pharm. 2012; 9(11):2961-2973.

17. Garanger E, Boturyn D, Dumy P. Tumor targeting with RGD peptide ligands - design of new molecular conjugates for imaging and therapy of cancers. Anticancer Agents Med Chem. 2007;7(5):552-558.

18. Zitzmann S, Ehemann V, Schwab M. Arginine-glycine-aspartic acid (RGD)-peptide binds to both tumor and tumor-endothelial cells in vivo. Cancer Res. 2002;62(18):5139-5143.

19. Maeda H, Wu J, Sawa T, Matsumura Y Hori K. Tumor vascular permeability and the EPR effect in macromolecular therapeutics: a review. J Control Release. 2000;65(1-2):271-284.

20. Yao Q, Gutierrez DC, Hoang NH, et al. Efficient codelivery of paclitaxel and curcumin by novel bottlebrush copolymer-based micelles. Mol Pharm. 2017;14(7):2378-2389.
21. Wei Y, Pu X, Zhao L. Preclinical studies for the combination of paclitaxel and curcumin in cancer therapy (Review). Oncol Rep. 2017;37(6): 3159-3166.

22. Trotta M, Debernardi F, Caputo O. Preparation of solid lipid nanoparticles by a solvent emulsification-diffusion technique. Int J Pharm. 2003; 257(1-2):153-160.

23. Kim SY, Lee YM. Taxol-loaded block copolymer nanospheres composed of methoxy poly(ethylene glycol) and poly(epsiloncaprolactone) as novel anticancer drug carriers. Biomaterials. 2001; 22(13):1697-1704.

24. Shi B, Fang C, You MX, et al. Stealth MePEG-PCL micelles: effects of polymer composition on micelle physicochemical characteristics, in vitro drug release, in vivo pharmacokinetics in rats and biodistribution in S180 tumor bearing mice. Colloid Polym Sci. 2005;283(9):954-967.

25. Turner EA, Stenson AC, Yazdani SK. HPLC-MS/MS method for quantification of paclitaxel from keratin containing samples. J Pharm Biomed Anal. 2017;139:247-251.

26. Malhi S, Stesco N, Alrushaid S, Lakowski TM, Davies NM, Gu X. Simultaneous quantification of reparixin and paclitaxel in plasma and urine using ultra performance liquid chromatography-tandem mass spectroscopy (UHPLC-MS/MS): application to a preclinical pharmacokinetic study in rats. $J$ Chromatogr B Analyt Technol Biomed Life Sci. 2017;1046:165-171.

27. Zheng Z, Sun Y, Liu Z, Zhang M, Li C, Cai H. The effect of curcumin and its nanoformulation on adjuvant-induced arthritis in rats. Drug Des Devel Ther. 2015;9:4931-4942.

28. Cao Y, Xu RX, Liu Z. A high-throughput quantification method of curcuminoids and curcumin metabolites in human plasma via highperformance liquid chromatography/tandem mass spectrometry. J Chromatogr B Analyt Technol Biomed Life Sci. 2014;949-950:70-78.
International Journal of Nanomedicine

\section{Publish your work in this journal}

The International Journal of Nanomedicine is an international, peerreviewed journal focusing on the application of nanotechnology in diagnostics, therapeutics, and drug delivery systems throughout the biomedical field. This journal is indexed on PubMed Central, MedLine, CAS, SciSearch $®$, Current Contents $\AA /$ Clinical Medicine,

\section{Dovepress}

Journal Citation Reports/Science Edition, EMBase, Scopus and the Elsevier Bibliographic databases. The manuscript management system is completely online and includes a very quick and fair peer-review system, which is all easy to use. Visit http://www.dovepress.com/ testimonials.php to read real quotes from published authors. 